\title{
QUALIDADE PÓS-COLHEITA DE ABACATES 'HASS' MANTIDOS EM TEMPERATURA AMBIENTE E TRATADOS COM DIFERENTES RADIAÇÕES
}

\author{
Juliana Wagner Simon ${ }^{1}$ \& Rogério Lopes Vieites ${ }^{2}$
}

\begin{abstract}
RESUMO: O presente trabalho teve como objetivo a conservação do abacate 'Hass' com uso da radiação. Foram realizados dois experimentos: Experimento I - frutos irradiados em diferentes doses de irradiação gama de cobalto 60 $(0,0 ; 0,2 ; 0,4 ; 0,6$ e 1,0 kGy); Experimento II - frutos irradiados por acelerador de elétrons em diferentes doses ( 0,0 ; 0,$48 ; 0,8 ; 1,12$ e $1,45 \mathrm{Mev}$ ) ambos mantidos sob temperatura ambiente $21 \pm 1^{\circ} \mathrm{C}$ e $70 \pm 5 \%$ UR. Foram realizadas análises de capacidade antioxidante, compostos fenólicos totais, perda de massa fresca e respiração a cada três dias, durante 12 dias. O delineamento experimental foi inteiramente casualizado, com três repetições por tratamento. Para análise estatística o Teste de Tukey a 5\% de probabilidade foi utilizado. Nas condições em que os experimentos foram realizados, os resultados permitiram concluir que o uso da radiação gama conservou os frutos por 12 dias independentes das doses aplicadas. O uso da radiação por acelerador de elétrons também favoreceu a conservação dos frutos independente da dose utilizada.
\end{abstract}

PALAVRAS-CHAVE: Persea americana Mill., radiação gama, acelerador de elétrons.

\section{POST-HARVEST QUALITY OF AVOCADO 'HASS' HELD IN AMBIENT TEMPERATURE AND TREATED WITH DIFFERENT RADIATION}

\begin{abstract}
This study aimed the avocado 'Hass' conservation with the use of radiation. We performed two experiments: Experiment I - fruits irradiated with different doses of cobalt-60 gamma irradiation $(0,0 ; 0,2 ; 0,4 ; 0,6$ e 1,0 $\mathrm{kGy})$; Experiment II - fruit irradiated by electron accelerator in different doses $(0,0 ; 0,48 ; 0,8 ; 1,12$ e $1,45 \mathrm{MeV})$, both maintained at room temperature of $21 \pm 1{ }^{\circ} \mathrm{C}$ and at relative humidity of $79 \pm 5 \%$. Antioxidant capacity, total phenolic compounds, fresh weight loss, and respiration rate evaluation were performed every three days for 12 days. The experimental design was completely randomized with three replicates per treatment. For statistical analysis, the Tukey test at $5 \%$ probability was employed. Under the conditions in which the experiments were performed, the results showed that the gamma radiation use retained the fruits for 12 days, regardless the doses applied. The radiation by electron accelerator use also promote the fruits preservation, regardless the doses employed.
\end{abstract}

KEYWORDS: Persea americana Mill., gamma radiation, electron accelerator.

\section{INTRODUÇÃO}

O abacate (Persea americana Mill.) é um fruto climatérico cujo amadurecimento ocorre poucos dias após colheita (HARDENBURG et al., 1986) com alta taxa respiratória e produção elevada de etileno. Sendo assim, torna-se importante controlar o amadurecimento desta fruta na tentativa de aumentar a vida-útil póscolheita, visando com isso o mercado interno e a exportação de frutas (BOWER; CUTTIN, 1988;

\footnotetext{
${ }^{1}$ E-mail: juwsimon@ gmail.com

${ }^{2}$ Faculdade de Ciências Agronomicas, Campus de Botucatu. E-mail vieites@fca.unesp.br
}

KADER, 1992; KLUGE et al., 2002; DAIUTO et al., 2010).

A refrigeração é o método de conservação pós colheita mais usado (BRON et al., 2002) e, a partir disso, novas técnicas têm sido estudadas para minimizar os efeitos do amadurecimento, entre elas a aplicação da radiação ionizante gama, tratamento térmico e mais recentemente radiação ultravioleta (DAIUTO et al., 2013). De acordo com Vieites (2009), a irradiação promete melhorar a habilidade na conservação dos frutos e reduzir a incidência de doenças sendo considerado um método seguro. Uma vantagem no uso desse método é que ele pode reduzir ou até substituir o uso de aditivos alimentares. 
Nos dias de hoje, a qualidade pós-colheita de um fruto pode estar relacionada com os benefícios que o mesmo possa trazer além de seu valor nutricional. O valor nutricional e comercial dos abacates 'Hass' justificam estudos de qualidade pós-colheita, assim como a determinação da capacidade antioxidante e compostos fenólicos e suas alterações com o armazenamento (DAIUTO et al., 2012).

Os antioxidantes são compostos que atuam inibindo e/ou diminuindo os efeitos desencadeados pelos radicais livres (SOARES et al., 2005), definidos como compostos que protegem as células contra os efeitos danosos dos radicais livres oxigenados e nitrogenados, formados nos processos oxidativos. Segundo Heim et al. (2002), os compostos fenólicos são os maiores responsáveis pela atividade antirradical livre em frutos, fazendo destes uma fonte natural de antioxidantes. A avaliação da capacidade antirradical livre tem sido importante para determinar a eficiência dos antioxidantes naturais em relação à proteção do produto vegetal contra dano oxidativo e perda do valor comercial e nutricional.

Desta maneira, a atual pesquisa teve como objetivo avaliar durante 12 dias a atividade antioxidante, compostos fenólicos, perda de massa fresca e atividade respiratória de abacates 'Hass' tratados por irradiação gama e por acelerador de elétrons em diferentes doses e mantidos sob temperatura ambiente $\left(21 \pm 1^{\circ} \mathrm{C}\right.$ e $70 \pm 5 \%$ UR).

\section{MATERIAL E MÉTODOS}

Foram utilizados frutos de abacate 'Hass', safra de 2010, produzidos em Bauru, São Paulo, Brasil, cujas coordenadas geográficas são: latitude 22¹9'18" S. longitude $49^{\circ} 04^{\prime} 13^{\prime \prime} \mathrm{W}$ e $526 \mathrm{~m}$ de altitude. Os frutos depois de colhidos no ponto de maturação fisiológica, com teor de óleo de $21.6 \%$, foram transportados ao laboratório e selecionados visando à homogeneização do lote quanto ao tamanho, cor e ausência de injúrias e defeitos. Em seguida, foram divididos em dois experimentos radiação gama de ${ }^{60}$ Co doses 0,$2 ; 0,4 ; 0,6$ e $1,0 \mathrm{kGy}$, e radiação por acelerador de elétrons doses 0,48; 0,80; 1,12 e 1,45 Mev.

\subsection{Análises}

Os frutos foram analisados aos $0,3,6,9$ e 12 dias de armazenamento, tempo este que os abacates mantiveram suas qualidades. Foram mantidos em temperatura ambiente $21 \pm 1^{\circ} \mathrm{C}$ e $70 \pm 5 \%$ de UR e, a cada tempo determinado para a análise, foram realizadas análises em triplicata, destrutivas e não destrutivas.

\subsubsection{Capacidade antioxidante e compostos fenólicos totais}

\subsubsection{Atividade antioxidante pelo método DPPH}

A medida da capacidade seqüestrante foi determinada pelo método DPPH (MENSOR et al., 2001). A atividade antirradical foi determinada na forma de atividade antioxidante (AA), pela equação:

$\mathrm{AA}(\%)=100-\{[(\mathrm{Aa}-\mathrm{Ab}) \times 100] / \mathrm{Ac}\}$
Onde:

Aa = absorbância da amostra;

$\mathrm{Ab}=$ absorbância do branco;

$\mathrm{Ac}=$ absorbância do controle negativo.

\subsubsection{Compostos fenólicos totais}

O conteúdo total de compostos fenólicos do extrato etanólico da polpa de abacate foi determinado pelo método espectrofotométrico de Folin-Ciocalteau (SINGLETON et al., 1999).

\subsubsection{Perda de massa fresca}

Para análise de perda de massa foi utilizada balança (Owalabor) com carga máxima de 2000 g e divisão de $10 \mathrm{mg}$.

\subsubsection{Respiração}

A curva de respiração foi obtida pela avaliação dos frutos a cada três dias, tanto para os abacates irradiados, quanto para os acelerados. A determinação da taxa de respiração feita de forma indireta foi efetuada em respirômetro, pela medida do $\mathrm{CO}_{2}$ liberado, de acordo com metodologia adaptada de Bleinroth et al. (1976). A taxa de respiração do abacate medida em respirômetro foi calculada pela seguinte fórmula:

$\mathrm{TCO}_{2}=\left[2.2 \times(\mathrm{A}-\mathrm{B}) \times \mathrm{V}_{1}\right] /\left(\mathrm{P} \times \mathrm{T} \times \mathrm{V}_{2}\right)$

Onde:

$\mathrm{TCO}_{2}=$ Taxa de respiração em $\mathrm{mL}$ de $\mathrm{CO}_{2} . \mathrm{Kg}$ de fruta 1 . hora $^{-1}$;

$\mathrm{B}=$ Volume gasto em $\mathrm{mL}$ de $\mathrm{HCl}$ padronizado para a titulação de hidróxido de potássio padrão antes da absorção de $\mathrm{CO}_{2}$;

$\mathrm{A}=$ Volume gasto de $\mathrm{HCl}$ padronizado para a titulação de hidróxido de potássio após a absorção de $\mathrm{CO}_{2}$ da respiração;

$\mathrm{V}_{1}=$ Volume de hidróxido de potássio usado na absorção de $\mathrm{CO}_{2}(\mathrm{~mL})$;

$\mathrm{P}=$ Peso dos frutos $(\mathrm{kg})$;

$\mathrm{T}=$ Tempo das reações metabólicas (1 hora);

$\mathrm{V}_{2}=$ Volume de hidróxido de potássio utilizado na titulação $(\mathrm{mL}) ; 2,2=$ devido ao equivalente de $\mathrm{CO}_{2}$ (44/2), multiplicado pela concentração do ácido clorídrico a $0,1 \mathrm{~N}$.

\subsubsection{Análise estatística}

O delineamento experimental foi inteiramente casualizado, fatorial 4 x 5(tratamento de radiação x dias de armazenamento). Os resultados da capacidade antioxidante e dos compostos fenólicos foram submetidos à análise de variância, seguida pelo teste de Tukey a 5\% de probabilidade.

\section{RESULTADOS E DISCUSSÃO}

\subsection{Experimento I - Radiação Gama $\left({ }^{60} \mathrm{Co}\right)$}

A tabela 1 apresenta os valores de capacidade antioxidante (\%) para polpas de abacates 'Hass' submetidos a diferentes doses de irradiação $(0,0 ; 0,2$; 0,$4 ; 0,6$ e 1,0 kGy) e armazenados em temperatura ambiente $\left(21 \pm 1^{\circ} \mathrm{C}\right.$ e $70 \pm 5 \%$ UR). 
Tabela 1 - Capacidade antioxidante (\%) por DPPH obtidos em abacates 'Hass' submetidos a diferentes


durante 12 dias (Botucatu. UNESP. 2011).

\begin{tabular}{cccccc}
\hline \multicolumn{7}{c}{ Dias de Armazenamento } \\
\hline Tratamento & $\mathbf{0}$ & $\mathbf{3}$ & $\mathbf{6}$ & $\mathbf{9}$ & $\mathbf{1 2}$ \\
\hline $\mathbf{0 , 0}$ kGy & 61,66 & 39,66 & 52,66 & 55,30 & 56,33 \\
$\mathbf{0 , 2} \mathbf{k G y}$ & 61,66 & 34,00 & 59,00 & 67,33 & 27,33 \\
$\mathbf{0 , 4}$ kGy & 61,66 & 35,66 & 58,00 & 62,33 & 45,33 \\
$\mathbf{0 , 6}$ kGy & 61,66 & 38,00 & 56,66 & 59,00 & 33,33 \\
$\mathbf{1 , 0}$ kGy & 61,66 & 66,33 & 47,66 & 41,00 & 55,33 \\
\hline Média & 51,94 & & &
\end{tabular}

Médias seguidas de mesma letra minúscula na coluna e maiúscula na linha não diferem entre si pelo Teste de Tukey a 5\% de probabilidade.

Os resultados da atividade antioxidante nas polpas de abacates 'Hass' submetidos a diferentes doses de irradiação não apresentaram diferenças estatísticas entre os dias de armazenamento e nem entre as doses de irradiação gama testadas. Neste trabalho a média geral encontrada para a capacidade antioxidante foi de $51,94 \%$, Pode-se observar que, ao final de 12 dias de armazenamento, a testemunha e frutos irradiados com dose 1,0 kGy foram as que obtiveram maior capacidade antioxidante ao longo do armazenamento. Daiuto et al. (2013) analisaram abacates 'Hass'tratados por UV-C durante $5,10,15$ e 20 minutos de exposição e notaram que os valores da capacidade antioxidante decresceram ao longo do armazenamento quando mantidos sob refrigeração $\left(10 \pm 1^{\circ} \mathrm{C}\right.$ e $90 \pm 5 \%$ UR).

Os valores de compostos fenólicos presentes nos abacates podem ser observados na tabela 2 .

Tabela 2 - Compostos fenólicos totais (mg ácido gálico $100 g^{-1}$ polpa) obtidos em abacates 'Hass' submetidos a diferentes doses de irradiação gama e armazenados em temperatura ambiente $\left(21 \pm 1^{\circ} \mathrm{C}\right.$ e $70 \pm 5 \%$ UR) durante 12 dias (Botucatu. UNESP. 2011).

\begin{tabular}{cccccc}
\hline \multicolumn{7}{c}{ Dias de Armazenamento } \\
\hline Tratamento & $\mathbf{0}$ & $\mathbf{3}$ & $\mathbf{6}$ & $\mathbf{9}$ & $\mathbf{1 2}$ \\
\hline $\mathbf{0 , 0} \mathbf{~ k G y}$ & 57,00 & 59,33 & 54,33 & 56,00 & 81,33 \\
$\mathbf{0 , 2} \mathbf{~ k G y}$ & 57,00 & 57,00 & 60,33 & 68,66 & 64,33 \\
$\mathbf{0 , 4} \mathbf{~ k G y}$ & 57,00 & 55,00 & 53,66 & 65,66 & 60,00 \\
$\mathbf{0 , 6} \mathbf{~ k G y}$ & 57,00 & 53,33 & 54,00 & 86,66 & 51,66 \\
$\mathbf{1 , 0} \mathbf{~ k G y}$ & 57,00 & 61,33 & 50,66 & 59,00 & 74,66 \\
\hline
\end{tabular}

Média

60,48

Médias seguidas de mesma letra minúscula na coluna e maiúscula na linha não diferem entre si pelo Teste de Tukey a 5\% de probabilidade.

Os resultados de compostos fenólicos totais não apresentaram diferenças estatísticas entre os dias de armazenamento, bem como nas doses aplicadas de radiação. A média geral apresentada neste experimento foi de $60,48 \mathrm{mg}$ ácido gálico $100 \mathrm{~g}^{-1}$ polpa. Daiuto et al. (2013) avaliaram abacates 'Hass' mantidos sob refrigeração $\left(10 \pm 1^{\circ} \mathrm{C}\right.$ e $90 \pm 5 \%$ UR $)$ e irradiados com UV-C em diferentes tempos de exposição 5, 10, 15 e 20 minutos não apresentaram diferenças entre os tratamentos dos frutos nos diferentes tempos de exposição.

A figura 1 apresenta a perda de massa fresca em abacates variedade 'Hass', armazenados em temperatura ambiente $\left(21 \pm 1^{\circ} \mathrm{C}\right.$ e $70 \pm 5 \%$ UR) e tratados com irradiação nas doses 0,$0 ; 0,2 ; 0,4 ; 0,6$ e $1,0 \mathrm{kGy}$. 


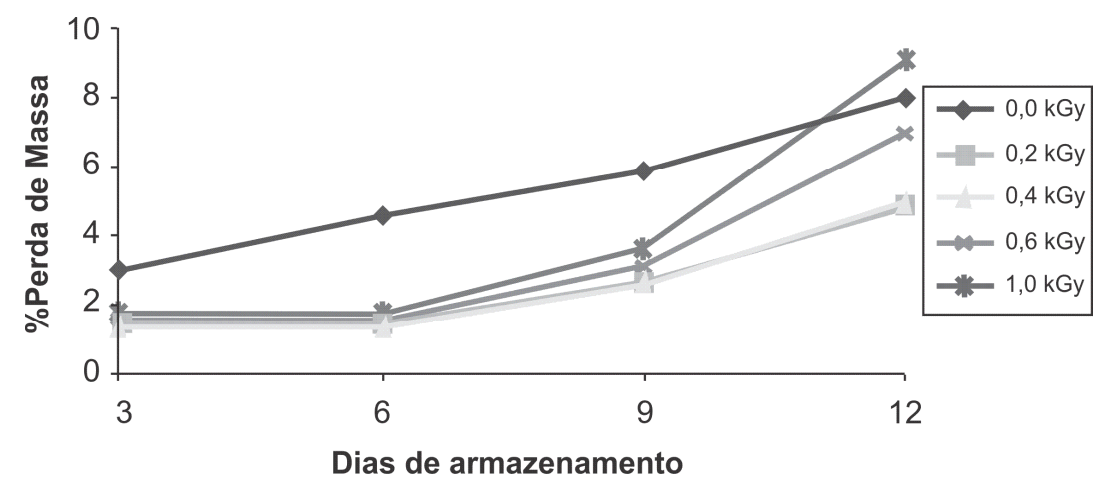

Figura 1 - Variação média de perda de massa fresca (\%) obtidos em abacates 'Hass' submetidos a diferentes doses de irradiação gama e armazenados em temperatura ambiente $\left(21^{\circ} \mathrm{C} \pm 1\right.$ e $70 \pm 5 \%$ UR) durante 12 dias (Botucatu. UNESP. 2011).

Os frutos controle e os irradiados apresentaram perda de massa fresca durante todo o decorrer do armazenamento. Verificou-se que até o nono dia de armazenamento, o controle foi o tratamento que apresentou maior perda de massa com o passar dos dias até o $12^{\circ}$ dia, onde se observou que a dose 1,0 kGy foi a que apresentou a maior perda de massa, que pode ser verificado talvez pelo fato dos frutos desse tratamento apresentarem maior transpiração. As doses 0,2 quanto e 0,4 kGy foram as que apresentaram menor perda de massa até o décimo segundo dia de armazenamento. Daiuto et al. (2010) também avaliaram a perda de massa em abacates 'Hass' tratados com diferentes doses de irradiação e armazenados sob temperatura ambiente e refrigeração, e notaram que a perda de massa não diferiu quanto às doses, porém apresentou diferenças quanto ao tipo de armazenamento, sendo que o refrigerado proporcionou menor perda de massa durante os dias de análises.

Na figura 2 observa-se a curva de respiração durante os 12 dias de armazenamento para os frutos de abacate 'Hass' tratados com diferentes doses de irradiação $(0,2$; $0,4 ; 0,6$ e $1,0 \mathrm{kGy})$.

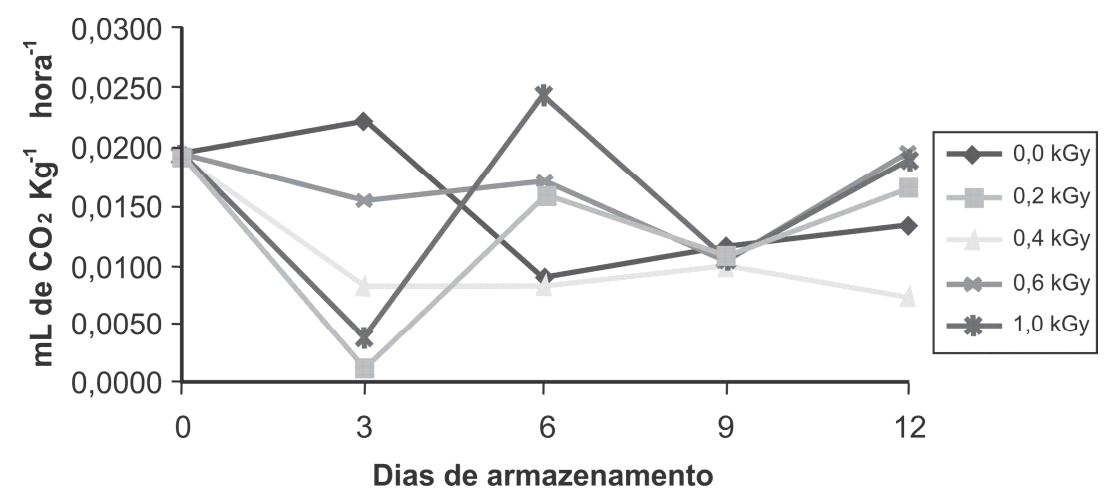

Figura 2 - Variação média da taxa respiratória ( $\mathrm{mL}$ de $\mathrm{CO}_{2} \mathrm{~kg}^{-1}$ hora ${ }^{-1}$ ) obtidos em abacates 'Hass' submetidos a diferentes doses de irradiação gama e armazenados em temperatura ambiente (21ํ \pm 1 e $70 \pm 5 \%$ UR) durante 12 dias (Botucatu. UNESP. 2011).

Notou-se neste experimento, que o controle obteve pico respiratório após três dias de armazenamento. As doses 0,2, 0,6 e 1,0 kGy apresentaram pico respiratório no sexto dia de armazenamento e a dose 0,4 kGy no nono dia. Sendo assim, para a taxa respiratória, a dose $0,4 \mathrm{kGy}$ foi a que apresentou melhor resposta a irradiação comparada ao controle. Daiuto et al. (2010) avaliaram abacates 'Hass' mantidos sob refrigeração e tratados com diferentes doses de irradiação e observaram que para a taxa respiratória a melhor dose aplicada foi a 0,2 kGy, dado esse diferente do encontrado na atual pesquisa. Molins (2001) relatou que os abacates são umas das frutas mais sensíveis a irradiação e que em muitas variedades doses maiores que 0,1 e $0,2 \mathrm{kGy}$ provocam manchas severas na pele bem como descolorações internas. Para muitas variedades, a dose ótima para retardar o amadurecimento e aumentar a vida comercial estão no intervalo entre 0,025 e 0,1 kGy. Já Vieites (2009), discordando de Molins (2001) relatou que para abacates a dose de irradiação favorável ao fruto é a 0,4 kGy, assim como encontrado neste trabalho.

\subsection{Experimento II - Acelerador de elétrons (Mega elétron volt - Mev)}

Verificou-se que para a capacidade antioxidante (Tabela 3) não houve diferenças estatísticas entre as doses aplicadas até o nono dia de armazenamento. Porem, no ultimo dia, pode-se notar que as doses $0,8,1,12$ e 1,45 Mev diferiram estatisticamente do controle e as doses 0,8 e $1,45 \mathrm{Mev}$ diferiram da dose $0,48 \mathrm{Mev}$. 
Tabela 3 - Capacidade antioxidante (\%) por DPPH obtidos em abacates 'Hass' submetidos a diferentes doses de irradiação e armazenados em temperatura ambiente $\left(21^{ \pm} 1^{\circ} \mathrm{C}\right.$ e $70 \pm 5 \%$ UR) durante 12 dias (Botucatu. UNESP. 2011).

Médias seguidas de mesma letra minúscula na coluna e maiúscula na linha não diferem entre si pelo Teste de Tukey a 5\% de probabilidade.

Neste experimento houve interação entre as doses de irradiação e os dias de armazenamento. No controle houve diferença estatística entre o primeiro, terceiro e nono dia de armazenamento. Para a dose 0,48 Mev, observou-se diferença estatística somente entre o dia zero e terceiro de armazenamento. Já para a dose 0,8 Mev, observou-se que a partir do dia 3 houve diferenças estatísticas quando comparado ao dia zero. Para a dose 1,12 Mev notou-se que somente no décimo segundo dia de armazenamento é que apresentou diferença em relação aos primeiros dias de análises. Para a dose 1,45 Mev verificou-se que o sexto dia de armazenamento diferiu do dia zero. Já para os demais dias, 3, 9 e 12, não houve diferença quando comparado ao primeiro dia de análise.

Nas condições deste experimento, verificou-se que não houve diferenças estatísticas para os compostos fenólicos totais entre as doses de radiação pelo acelerador de elétrons aplicadas aos frutos durante os doze dias de armazenamentos. Diferença estatística pode ser observada para a dose $1,12 \mathrm{Mev}$, onde o $9^{\circ}$ dia diferiu estatisticamente do $12^{\circ}$ dia de armazenamento. Notou-se nesta pesquisa que o tratamento controle apresentou aumento dos compostos fenólicos totais no décimo segundo dia de armazenamento. Fato semelhante pode ser observado pela dose 1,45 Mev aplicada aos frutos. Trabalhos realizados por Daiuto et al. (2013) mostraram que abacates 'Hass' armazenados sob refrigeração $\left(10 \pm 1^{\circ} \mathrm{C}\right.$ e $90 \pm 5 \%$ UR) e tratados por UV-C $(5,10,15$ e 20 minutos de exposição) não apresentaram diferenças entre os dias de armazenamento. Neste experimento, as doses $0,48,0,8$ e $1,12 \mathrm{Mev}$ apresentaram maiores valores de compostos fenólicos totais no nono dia de armazenamento, sendo que o maior valor encontrado foi de $86,66 \mathrm{mg}$ ácido gálico $100 \mathrm{~g}$-1 polpa para a dose 1,12 Mev (Tabela 4).

Tabela 4: Compostos fenólicos totais (mg ácido gálico 100g-1 polpa) obtidos em abacates 'Hass' submetidos a diferentes doses de irradiação por acelerador de elétrons e armazenados em temperatura ambiente $\left(21 \pm 1^{\circ} \mathrm{C}\right.$ e $70 \pm 5 \%$ UR) durante 12 dias (Botucatu. UNESP. 2011).

\begin{tabular}{cccccc}
\hline \multicolumn{7}{c}{ Dias de Armazenamento } \\
\hline Tratamento & $\mathbf{0}$ & $\mathbf{3}$ & $\mathbf{6}$ & $\mathbf{9}$ & $\mathbf{1 2}$ \\
\hline $\mathbf{0 , 0} \mathbf{M e v}$ & $57,00 \mathrm{aA}$ & $59,33 \mathrm{aA}$ & $54,33 \mathrm{aA}$ & $56,00 \mathrm{aA}$ & $81,33 \mathrm{aA}$ \\
$\mathbf{0 , 4 8} \mathbf{~ M e v}$ & $57,00 \mathrm{aA}$ & $57,00 \mathrm{aA}$ & $60,33 \mathrm{aA}$ & $68,66 \mathrm{aA}$ & $64,33 \mathrm{aA}$ \\
$\mathbf{0 , 8} \mathbf{~ M e v}$ & $57,00 \mathrm{aA}$ & $55,00 \mathrm{aA}$ & $53,66 \mathrm{aA}$ & $65,66 \mathrm{aA}$ & $60,00 \mathrm{aA}$ \\
$\mathbf{1 , 1 2} \mathbf{~ M e v}$ & $57,00 \mathrm{aAB}$ & $53,33 \mathrm{aAB}$ & $54,00 \mathrm{aAB}$ & $86,66 \mathrm{aA}$ & $51,66 \mathrm{aB}$ \\
$\mathbf{1 , 4 5} \mathbf{M e v}$ & $57,00 \mathrm{aA}$ & $61,33 \mathrm{aA}$ & $50,66 \mathrm{aA}$ & $59,00 \mathrm{aA}$ & $74,66 \mathrm{aA}$ \\
\hline Média & 60,48 & & &
\end{tabular}

Médias seguidas de mesma letra minúscula na coluna e maiúscula na linha não diferem entre si pelo Teste de Tukey a $5 \%$ de probabilidade.

A Figura 3 evidencia a perda de massa fresca dos frutos irradiados por acelerador de elétrons em diferentes doses durante 12 dias de armazenamento em condições de temperatura ambiente $21 \pm 1^{\circ} \mathrm{C}$ e $70 \pm 5 \%$ UR. 


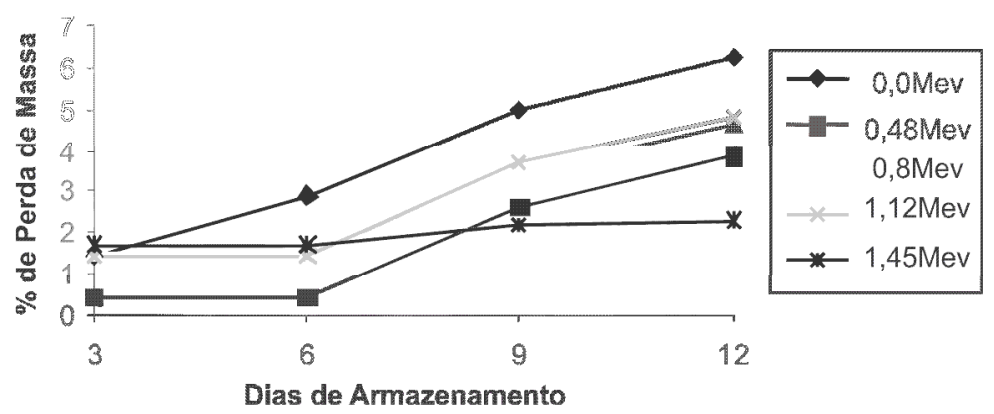

Figura 3 - Variação média de perda de massa fresca (\%) obtidos em abacates 'Hass' submetidos a diferentes doses de irradiação por acelerador de elétrons e armazenados em temperatura ambiente $\left(21 \pm 1{ }^{\circ} \mathrm{C}\right.$ e $70 \pm 5 \%$ UR) durante 12 dias (Botucatu. UNESP. 2011).

Neste experimento, observou-se que para todos os frutos que receberam tratamento de irradiação através de acelerador de elétrons obtiveram pico respiratório após 6 dias de armazenamento (Figura 4). Fato semelhante a este também pode ser observado para o controle que obteve seu pico respiratório após 6 dias de armazenamento. Verificou-se que o controle foi $o$ tratamento que apresentou a maior taxa respiratória em relação às doses aplicadas.

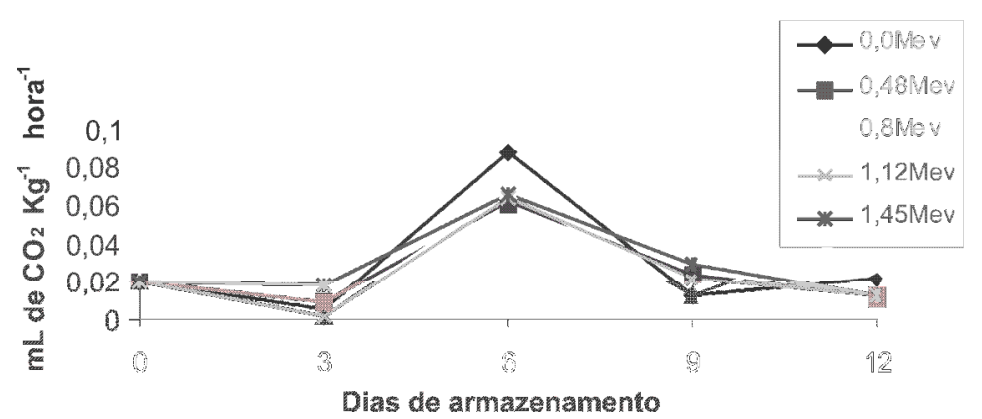

Figura 4 - Variação média da taxa respiratória ( $\mathrm{mL}$ de $\mathrm{CO}_{2} \mathrm{~kg}^{-1}$ hora ${ }^{-1}$ ) obtidos em abacates 'Hass' submetidos a diferentes doses de irradiação por acelerador de elétrons e armazenados em temperatura ambiente $\left(21 \pm 1^{\circ} \mathrm{C}\right.$ e $70 \pm 5 \%$ UR) durante 12 dias (Botucatu. UNESP. 2011).

Para as diferentes doses de irradiação, verificou-se que os picos climatéricos ficaram bem próximos em relação à taxa respiratória. Daiuto et al. (2010) afirmaram que a intensidade da taxa respiratória está relacionada com a capacidade de armazenamento do produto, e que, quanto maior a taxa respiratória, menor é o tempo de armazenamento.

\section{CONCLUSÃO}

Nas condições em que os experimentos foram realizados, as análises dos resultados permitiram concluir que tanto a radiação gama quanto a radiação por acelerador de elétrons controlaram o amadurecimento dos frutos, independentes das doses aplicadas, por 12 dias, tempo este que os frutos entraram em senescência.

\section{REFERÊNCIAS}

BLEINROTH, E. W.; ZUCHINI, A. G.; POMPEO, R. M. Determinação das características físicas e mecânicas de variedade de abacate e sua conservação pelo frio. Coletânea ITAL, Campinas, v. 7, n. 1, p. 29-81, 1976.
BOWER, J.; CUTTING, J. G. Avocado fruit development and ripening physiology. Horticultural Review, London, v. 10, p. 229-271, 1988.

BRON, I. U.; JACOMINO, A. P.; APPEZZATO-DAGLORIA, B. Alterações anatômicas e físico-químicas associadas ao armazenamento refrigerado de pêssegos 'Aurora-1' e 'Dourado-2 '. Pesquisa Agropecuária Brasileira, Brasília, DF, v. 37, n. 10, p. 1349-1358, 2002.

DAIUTO, E. R. et al. Taxa respiratória de abacate 'Hass' submetido a diferentes tratamentos físicos. Revista Iberoamericana de Tecnología Postcosecha, Hermosillo, México, v. 10, n. 2, p. 101-109, 2010.

DAIUTO, E. R. et al. Capacidade anti radical livre e qualidade pós colheita de abacate 'Hass'. Revista Brasileira de produtos Agroindustriais, Campina Grande, v. 14, n. 1, p. 51-62, 2012.

DAIUTO, E. R. Pós colheita do abacate 'Hass'submetido a radiação UV-C. Revista Colombiana de Ciências Hortícolas, Bogotá, v. 7, n. 2, p. 149-160, 2013. 
HARDENBURG, R. E.; WATADA, A. E.; WANG, C. $Y$. The commercial storage of fruits. vegetables and florist and nursery stocks. Washington, DC: USDA, 1986. 130 p. (Agriculture Handbook, 66).

HEIM, K. E.; TAGLIAFERRO, A. R.; BOBILYA, D. J. Flavonoid antioxidants: chemistry, metabolism ande structure activity relationships. Reviews: current topics. The Journal of Nutritional Biochemistry, Stoneham, v. 13, p. 572-584, 2002.

KADER, A. A. Postharvest biology and technology: an overview. In: __ Postharvest technology of horticultural crops. 2. ed. Davis: University of California, 1992. 295 p.

KLUGE, R. A. et al. Inibição do amadurecimento de abacate com 1-metilciclopropeno. Pesquisa Agropecuária Brasileira, Brasília, DF, v. 37, n. 7, p. 895-901, 2002.

MENSOR, L. L. et al. Screening of Brazilian plant extracts for antioxidant activity by the use of DPPH free radical method. Phytotherapy Research, London, v. 15, p. 127-130, 2001.

MOLINS, R. Introducción. In: Irradiación de alimentos: principios y aplicaciones. Zaragosa: Acribia, 2001. p. 1-20.

SINGLETON, V. L.; ORTHOFER, R.; LAMUELA, R. M. Analysis of total phenols and other oxidation substrates and antioxidants by means of Folin-Ciocalteau reagent. Methods of Enzymology, New York, v. 299, p. 152-178, 1999.

Soares, D. G.; Andreazza, A. C.; Salvador, M. Avaliação de compostos com atividade antioxidante em células de levedura Saccharomyces cerevisiae. Revista Brasileira de Ciências Farmacêuticas, Araraquara, v. 41, n. 1, p. 95-100, 2005.

VIEITES, R. L. Conservação pós-colheita de frutos com a utilização de irradiação. In: NEVES, L. C. (Org.). Manual pós-colheita da fruticultura brasileira. Londrina: Eduel, 2009. p. 189-212. 